\title{
Other and III-Defined Sites in Lip, Oral Cavity and Pharynx ICD-O-3
}

National Cancer Institute

\section{Source}

National Cancer Institute. Other and III-Defined Sites in Lip, Oral Cavity and Pharynx ICD-

0-3. NCI Thesaurus. Code C148337.

Anatomic sites in the lip, oral cavity and pharynx that are referenced in topographic category C14 of ICD-O-3. 